\title{
Objetos metálicos del Cerro del Bu (Toledo) ${ }^{1}$
}

\author{
Jacobo Fernández del Cerro
}

\section{Resúmen}

En el siguiente trabajo se presentan una serie de materiales metálicos pertenecientes al yacimiento toledano de El Cerro del Bu, uno de los escasos ejemplos excavados de ocupación de carácter permanente la Edad del Bronce en la Cuenca Media del Tajo. Estos objetos ponen de manifiesto el desarrollo en la zona de una metalurgia de carácter doméstico, aunque no documentada en el yacimiento, que se ve complementada con la presencia de algunos objetos de prestigio como un puñal con remaches de plata o una cinta de oro que evidencian la existencia de una clase dirigente posiblemente apoyada en el control de los recursos agropecuarios y de las vías de comunicación.

\begin{abstract}
This work presents several metal objets of the Cerro del Bu site. This site is one of the few Bronze Age long-term settlements excavated in the middle Tagus Valley. These materials show the development of a domestic metallurgical production although it is not documented in the site. The discovery of prestige objetcs, such as a rivetted dagger with a silver rivet or a gold strip, indicates the existence of a leading class based on the control of the farming resources and the communication routes.
\end{abstract}

El yacimiento del Cerro del Bu se localiza frente al casco histórico de Toledo, en la orilla izquierda del Tajo (Figura 1). Se trata de una pequeña elevación situada sobre el río donde se desarrollaron en los años 80 una serie de excavaciones que se prolongaron durante toda la década y cuyos resultados fueron expuestos en el I Congreso de Arqueología de la Provincia de Toledo (de Álvaro y Pereira, 1990). En este yacimiento, con una larga ocupación durante el Bronce Antiguo, se documentaron cabañas de planta circular u oval con zócalo de piedra y paredes y techos de ramaje que se disponían sobre aterrazamientos artificiales realizados mediante muros de mampostería en la ladera meridional del cerro.

A partir del estudio de la cerámica del Corte 6 se pueden identificar al menos cuatro fases en el asentamiento:

- Una primera perteneciente a los momentos iniciales del Bronce Antiguo, relacionada con las dataciones radiocarbónicas realizadas que proporcionaron fechas muy antiguas: I-13959: $3970 \pm 100$ B. P. y I-14416: $3830 \pm 100$ B. P. (de Álvaro y Pereira, 1990: 205), y que en fechas calibradas se elevan a 2492 y 2315 cal. B. C. (Castro y otros, 1996). El material cerámico no es muy abundante y está caracterizado por la presencia de formas de tendencia esférica y perfiles en $\mathrm{S}$ acompañados de algunos vasos carenados (Figura 2). Las decoraciones se reducen a cordones, digitaciones e impresiones de punzón sobre el borde, mientras que entre los tratamientos superficiales sobresalen los espatulados. En este paquete estratigráfico se halló un fragmento con decoración tipo Dornajos. La aparición en los niveles superficiales de fragmentos de campaniforme puntillado debe estar relacionada también con este momento.

- Una segunda etapa correspondiente a una fase evolucionada del Bronce Antiguo, que quizás enlazaría con los inicios del Bronce Medio y a la que debe estar asociado el puñal de remaches. En esta etapa abundan las formas carenadas de diferentes tamaños, caracterizadas por los acabados bruñidos o espatulados (Figura 3), con paralelos en algunos yacimientos madrileños como Tejar del Sastre (Quero, 1982). Las decoraciones no son muy distintas a la fase anterior aunque ahora destacan algunos

1. Agradezco a la doctora Concepción Blasco Bosqued y al doctor Juan Pereira Sieso la ayuda prestada para la realización de este trabajo. 

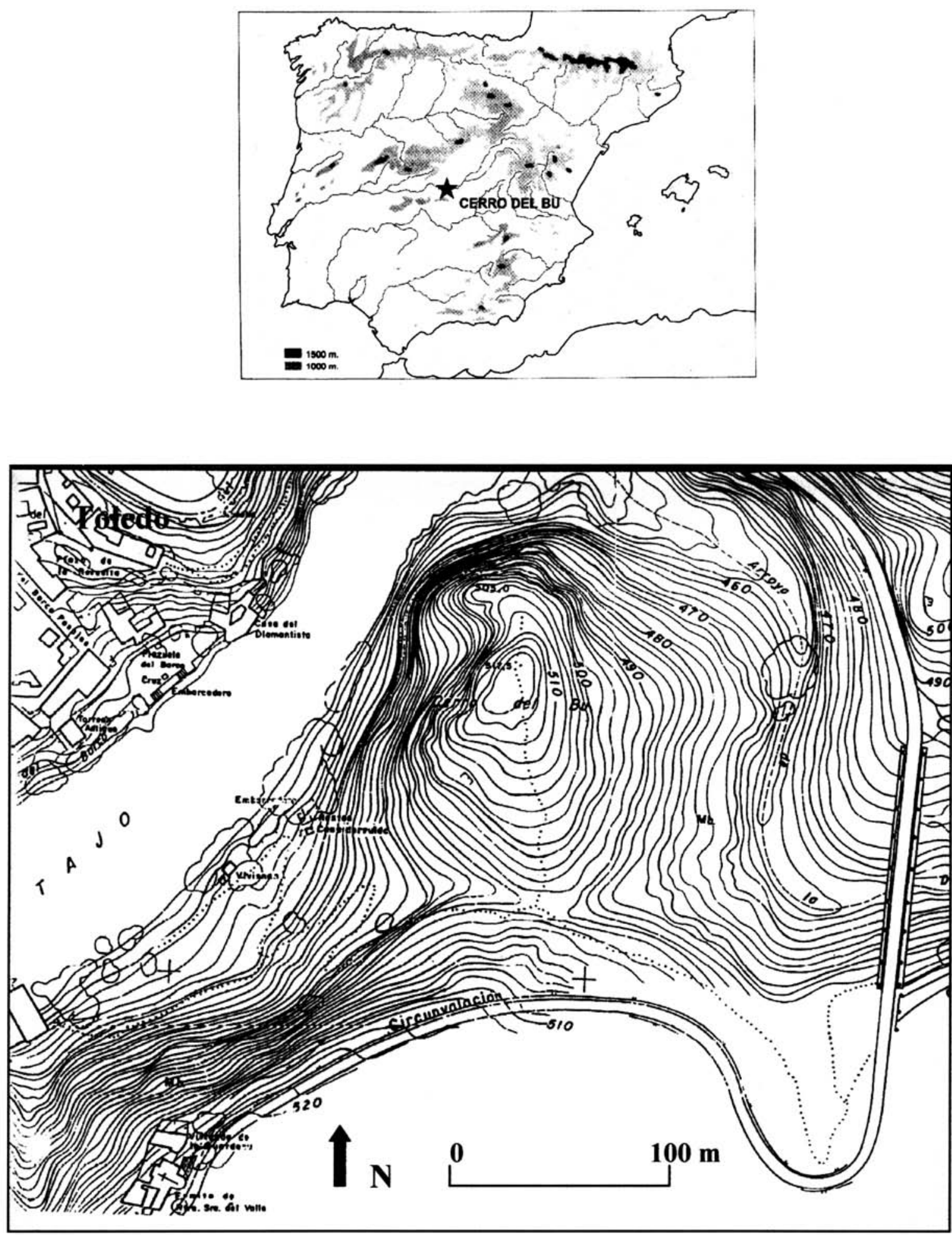

Figura 1.- Plano de localización del Cerro del Bu. 


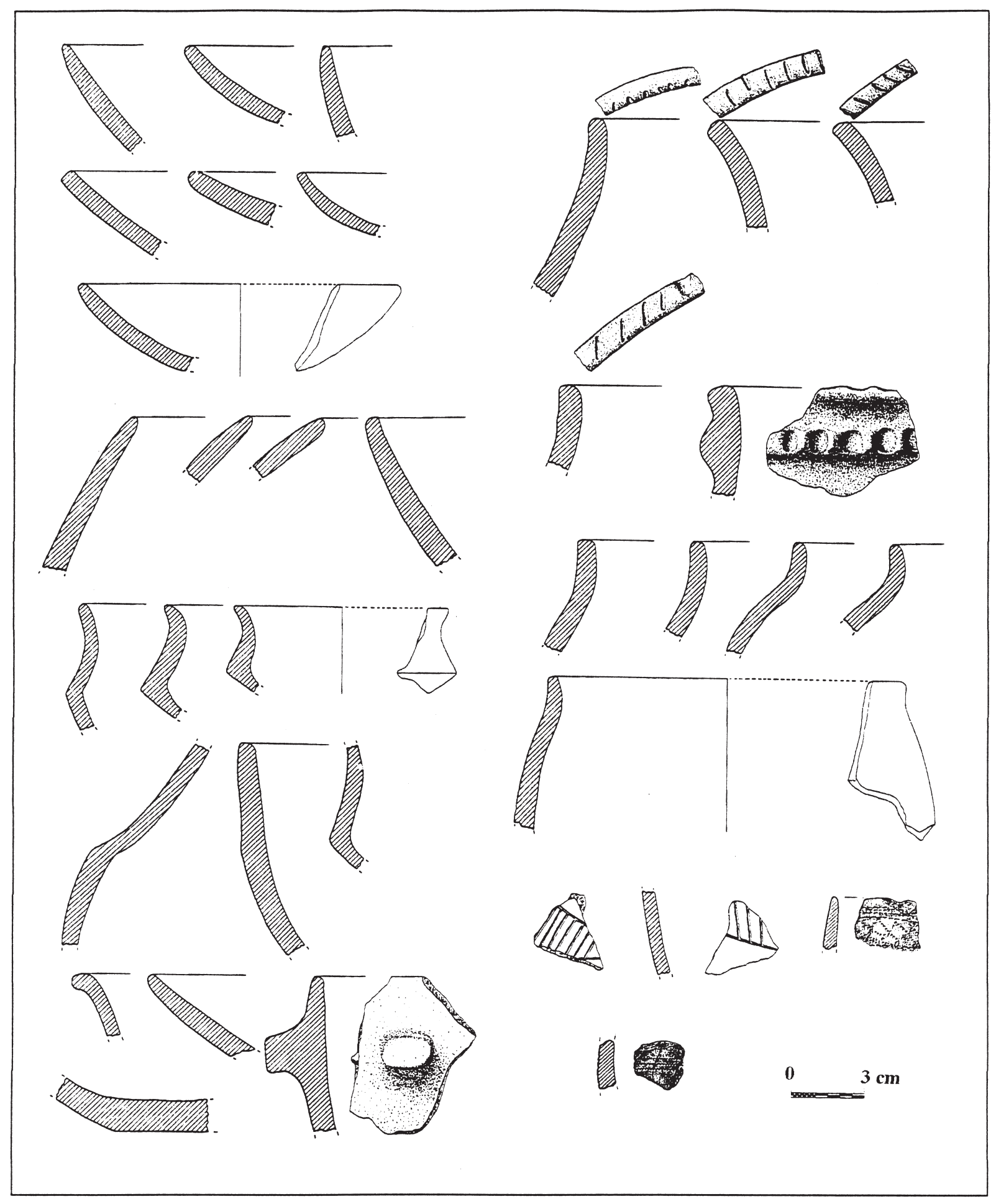

Figura 2.- Cerámica. Primera fase del yacimiento. 


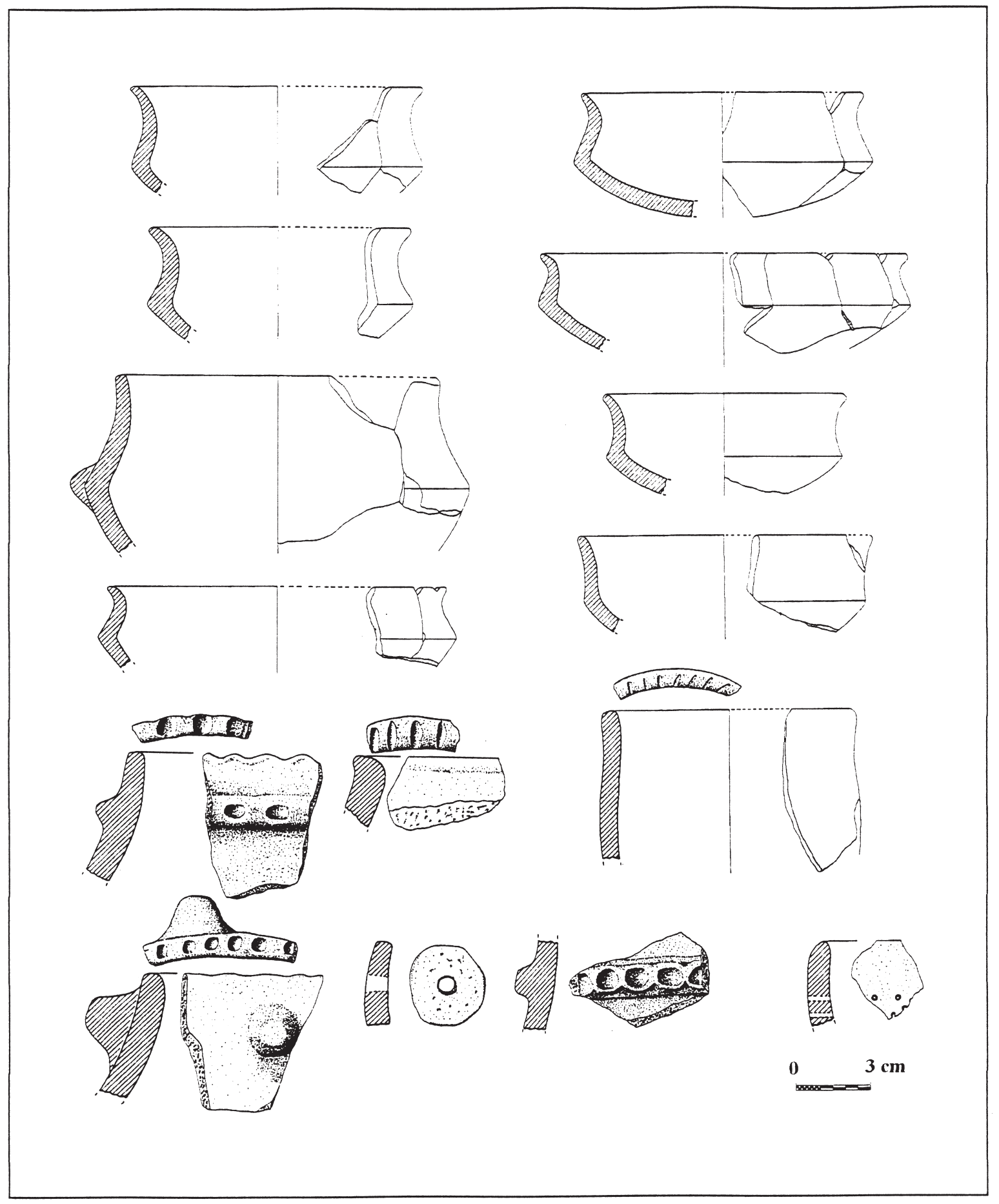

Figura 3.- Cerámica. Segunda fase del yacimiento 
cordones digitados aplicados formando círculos o ramificaciones.

- Una tercera etapa escasamente representada que se podría encuadrar en el Hierro Antiguo (siglo VIII-VII a. C.) con algunos elementos que recuerdan a Cogotas I y a los Campos de Urnas. Las carenas se suavizan y presentan un menor diámetro que las bocas de los recipientes. Aparecen los acabados escobillados en la cerámica menos cuidada y los espatulados dominan entre la cerámica fina. Encontramos mamelones perforados, decoraciones de hoyitos digitados, algún fragmento acanalado, cordones impresos y zigzags incisos.

- Una cuarta fase medieval islámica datable en el siglo X d. C., momento en el que se construye una pequeña fortificación sobre la parte superior del cerro.

\section{Panorama general de la metalurgia EN LA MESETA SUR EN EL BRONCE ANTI- GUO Y MEDIO}

La importancia de la metalurgia en la Meseta Sur va a ser considerada como secundaria ya que se trata una producción escasa y no especializada (Fernández Posse y otros, 1999: 218). Esta zona se encuentra en la Edad del Bronce en un nivel tecnológico muy similar a otras regiones peninsulares a pesar de la tradicional visión de ser una región marginal de los grandes centros de producción de metal (Blasco y Rovira, 1992-1993: 398). Estamos ante una metalurgia de pequeña escala destinada a satisfacer las necesidades domésticas. La producción se centra en piezas de pequeño tamaño y morfología sencilla con fines utilitarios.

Durante el Calcolítico precampaniforme los objetos metálicos son excepcionales, aparecen formando parte de ajuares funerarios de personajes de alto rango y no está comprobada su utilización en ambientes domésticos.

Los primeros elementos contextualizados que evidencian actividad metalúrgica en la Cuenca Media del Tajo se encuentran asociados al Horizonte Campaniforme. En esta etapa se puede encuadrar el yacimiento madrileño de El Ventorro donde se han recuperado crisoles, vasijas horno y goterones de fundición, así como el toledano yacimiento de El Guijo donde se halló un fragmento de crisol con adherencias de cobre (Rojas y Rodríguez, 1990: 175). Algunos investigadores hablan de generalización de la metalurgia durante esta etapa al aumentar el número de piezas y tener un mejor conocimiento de su pro- ceso de fabricación (Blasco y Rovira, 1992-1993: 401).

Durante el Bronce Antiguo se conserva la tradición metalúrgica campaniforme en el trabajo exclusivo del cobre y en la tipología de las piezas (Blasco y otros, 1995 a: 116). Es común en estas primeras etapas, la aparición de cobres arsenicales propios de la Península Ibérica durante el Calcolítico y Bronce Antiguo.

A partir del Bronce Pleno encontramos los primeros bronces binarios asociados a cerámicas lisas, como en Tejar del Sastre y La Loma del Lomo, y a contextos protocogotas, como en el Caserío de Perales, donde también se han hallado indicios de actividad metalúrgica (Blasco y otros, 1995 a: 118). Esta prematura metalurgia de Bronce que se da en los grupos protocogotas, ha llevado a proponer la hipótesis de que éstos pudieron introducir el bronce entre los fundidores del Sur, que ya habían logrado espectaculares producciones en cobre destinadas a obtener elementos de prestigio, mientras que los pequeños talleres meseteños estaban especializados en la producción de sencillos objetos de uso cotidiano de gran calidad con fines únicamente funcionales (Blasco y Rovira, 1992-1993: 402). Esta sospecha se ve reforzada por la confirmación de que la difusión de la aleación de bronce se produce de Norte a Sur tardando al menos dos siglos en extenderse desde el tercio Norte, al resto del territorio peninsular (Fernández Miranda y otros, 1995: 58). Estos primeros bronces con bajo contenido en estaño $(<10 \%)$ aparecerán en Navarra, Galicia y el Valle del Ebro en torno al primer cuarto del II milenio a. C, llegando a la Cuenca Media del Tajo probablemente a partir del 1500 a. C. En La Loma del Lomo (Guadalajara) se recuperó un punzón de bronce en un contexto que se puede fechar en $1420 \pm 100$ a. C. (Fernández Miranda y otros, 1995: 64) y, encuadrados en la fase protocogotas del Caserío de Perales (Madrid), también se han hallado elementos realizados en bronces binarios con una presencia de estaño entre el 8 y el $20 \%$ que han sido fechados en 1629 cal. B.C. (Blasco y otros, 1995 b).

El proceso de adopción del bronce es muy lento y se ha justificado por la existencia de grupos culturales intermedios que actúan como filtros de las novedades. A juzgar por los datos disponibles, cuando en el Valle del Tajo ya se ha consolidado la aleación cobre-estaño, La Mancha, la costa Levantina, el Sureste y las Baleares presentan una total ausencia de signos metálicos modernos, lo que evidencia un cierto aislamiento tecnológico (Fernández Miranda y otros, 1995: 67). Este aislamiento quizá se debe al 
carácter esporádico y a la baja intensidad de los contactos con la Meseta central y septentrional y la zona Norte de la Península.

Es posible que la adición de estaño esté en función de las disponibilidades, no dependiendo tanto de las mejoras que aporta la aleación. El hecho de que los bronces más ricos aparezcan en las zonas con recursos propios de estaño parece avalar esta hipótesis (Fernández Miranda y otros, 1995: 66).

\section{LOS RECURSOS MINERALES}

Se conocen escasos testimonios de minería prehistórica en toda la Península Ibérica por lo que la mayoría de los estudios se han centrado en la potencialidad de las diferentes regiones.

En la Meseta Sur parece darse una disposición periférica de los recursos de cobre concentrados en los rebordes montañosos de la penillanura (Fernández Posse y otros, 1999: 221). El problema que se debió dar en la Edad del Bronce por la relativa lejanía de los recursos minerales de cobre, parece perfectamente superable más aún cuando hay documentados largos desplazamientos para la obtención de materias primas destinadas a la fabricación de instrumentos líticos y si tenemos en cuenta, además, el pequeño volumen de mineral que requeriría la producción metalúrgica en estos momentos (Fernández Posse y otros, 1999: 221). Hay que señalar que en estas primeras etapas metalúrgicas, las actividades de reducción del mineral de cobre se suelen realizar en los poblados hasta donde se transporta el mineral en bruto (Montero, 1992: 194). El hallazgo de un metapodio de buey deformado en la excavación del Sector III de Getafe (Blasco y Barrio, 1986) ha sido interpretado como una evidencia de su utilización como animal de carga, sistema que pudo ser el empleado en el área madrileña para el traslado de materias primas como el granito o el mineral de cobre varias decenas de kilómetros desde el lugar de aprovisionamiento en la Sierra, hasta los asentamientos situados en las terrazas bajas de los ríos (Blasco y Rovira, 1992-1993: 401).

En el área toledana, Ruiz Taboada y Montero dudan de la existencia de un sistema de intercambio aplicable a los metales en la Edad del Bronce (Ruiz Taboada y Montero, 2000: 364). Estos autores prefieren proponer una movilidad a pequeña escala de los grupos de esta zona relacionada con una trashumancia de corto alcance que propiciase desplazamientos de hasta 40 kilómetros, aprovechados para el aprovisionamiento de metal o piedra.
La Provincia de Toledo posee una enorme riqueza minera, en especial la zona Sur de la Provincia, ocupada por las estribaciones septentrionales de los Montes de Toledo (Montero y otros, 1990). Sin embargo, pese a esta relativa concentración de mineral de cobre, se han rebatido las hipótesis que consideraban a Los Montes de Toledo como un importante centro de producción de metal durante la Edad del Bronce (Ruiz Taboada y Montero, 2000: 357). El modelo que propone Montero (1992) para explicar la metalurgia del Sureste peninsular durante el Bronce Antiguo y Pleno, es aplicable perfectamente en la Cuenca Media del Tajo donde la metalurgia sería una actividad secundaria desde el punto de vista económico. Pese a ser una producción de carácter doméstico, pudo producirse algún intercambio de objetos aunque lejos de alcanzar una producción comercializada.

En los Montes de Toledo son comunes las mineralizaciones complejas de cobre en las que aparece asociado con otros metales como el estaño o el bismuto. La comparación de la composición de los minerales disponibles con la de las manufacturas puede ofrecer datos sobre la procedencia de la mena de cobre utilizada, sobre todo cuando la simplicidad tecnológica facilita que el metal conserve mejor el cuadro de impurezas del mineral de partida (Blasco y Rovira, 1992-1993: 406). Pero también hay que tener en cuenta el comportamiento en el proceso de producción de muchos elementos presentes en la composición del cobre metálico, que puede cambiar al ser sometido a aumentos de temperatura y tratamientos mecánicos (Fernández Posse y otros, 1999: 223).

En el Cerro del Bu no se conoce ningún dato acerca de los procesos de extracción y de las actividades de transformación y producción de los elementos metálicos y no se ha hallado hasta el momento ningún resto u objeto utilizado en el proceso como crisoles o vasijas horno donde se realizaba la reducción del mineral, ambos bien documentados en el área madrileña (Rovira y Montero, 1994). Únicamente el hallazgo de una bolita de metal (Figura 6.3) en los niveles correspondientes a la segunda fase del yacimiento se puede relacionar con la práctica de operaciones de fundición de cobre. En la provincia de Toledo, además del fragmento de crisol antes mencionado de El Guijo, se han hallado restos de fundición en Silos de La Atalaya y fragmentos de vasijahorno en El Fontarrón (Montero, 2001: 284).

\section{LOS MATERIALES}

La totalidad de las piezas que se exponen a 
continuación proceden de las excavaciones realizadas en el Cerro del Bu. Sin embargo, no todas se pueden situar en un contexto claro, ya que muchas de ellas aparecen en los niveles superficiales o en otros revueltos junto a materiales del Hierro I. La primera etapa del yacimiento, correspondiente a un primer momento del Bronce Antiguo, presenta un punzón como único elemento contextualizado (Figura 5.2), y, como veremos, la mayoría de los hallazgos se podrían adscribir a la segunda fase del mismo.

Antes de comenzar la descripción de las piezas hay que señalar que la utilización de elementos tipológicos como criterio de datación se enfrenta al problema de la perduración y coexistencia de tipos que impide asignar intervalos cronológicos fiables (Fernández Miranda y otros, 1995: 59). También hay que tener en cuenta al establecer paralelos tipológicos que resulta difícil aplicar en un área periodizaciones de otras zonas donde las manufacturas se encuadran y funcionan de forma más ajustada.

\section{El puñal (Figura 4)}

El puñal del cerro del $\mathrm{Bu}$ tiene una longitud de $15 \mathrm{~cm}$ y una anchura media de $1,5 \mathrm{~cm}$ en el extremo distal y $2,2 \mathrm{~cm}$ en la zona de enmangue.
Esta última, muestra una forma ligeramente convexa donde se disponen formando un triángulo tres remaches, de los cuales se conserva uno realizado en plata y el inferior realizado en cobre, mientras que del otro, perdido, se mantiene la perforación circular fracturada donde se alojaba. Los remaches presentan una cabeza circular plana y un vástago de sección también circular. Los filos son casi paralelos, la punta redondeada y la hoja presenta una sección lenticular aplanada. No se observan huellas ni restos del enmangue orgánico que debió cubrir el extremo proximal de la pieza. La hoja se conserva en buen estado gracias al tratamiento de restauración realizado, aunque la punta está ligeramente doblada. Los filos no presentan huellas de utilización a simple vista, aunque se encuentran algo dentados posiblemente debido a procesos postdeposicionales.

Este tipo de puñal de hoja tan estilizada no es muy común y son escasos los paralelos encontrados, ninguno de ellos en la Meseta. Aparece asociado a contextos argáricos avanzados como en Peñalosa (Moreno Onorato, 2000: 204) con una cronología entre 2042 y 1690 cal. B.C. Encontramos, también en el valle del Guadalquivir, un puñal muy similar, aunque algo mayor (23 cm de longitud), en una sepultura del

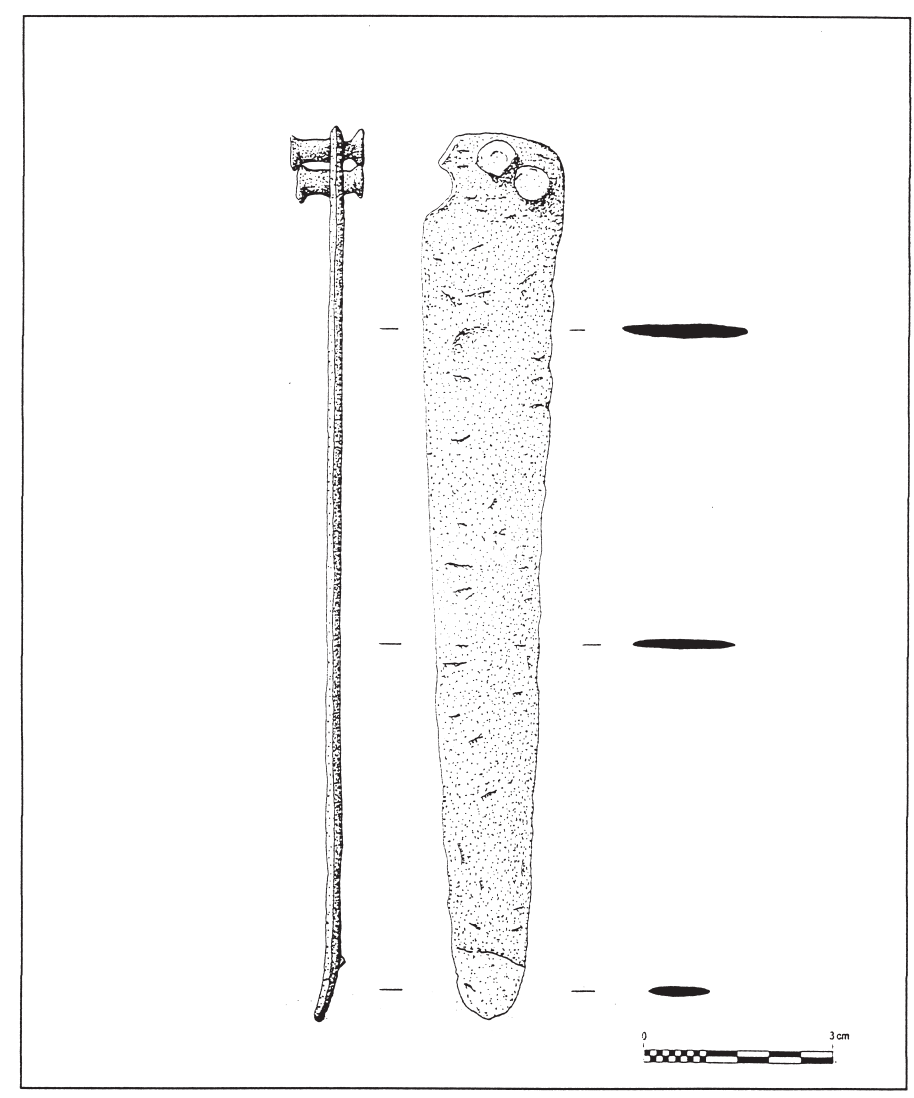

Figura 4.- Puñal de remaches. 
estrato XIV de Setefilla (Lora del Rio, Sevilla) perteneciente a una fase de transición entre el Bronce Antiguo y Pleno del yacimiento y que ha proporcionado la fecha de $1859 \mathrm{cal}$. ane. (Aubet y Serna, 1981: 243; Castro y otros, 1996). Aubet y Serna identifican el puñal de Setefilla con el Tipo I de Blance (puñales largos asociados a enterramientos en pithos característicos del Argar b) aunque la disposición triangular de los remaches, y la placa de enmangue lo relacionen con puñales del tipo III algo más antiguos (Aubet y Serna, 1981: 243; Blance, 1971: 124-125, lam. 23, no 4). Estas piezas son características del Alto Alentejo y Huelva en el Bronce Medio (Aubet y Serna, 1981: 244).

Quizás el puñal del Cerro del Bu deba ser considerado como una pieza fabricada fuera del asentamiento. Los objetos metálicos encontrados en otros yacimientos de la Cuenca Media del Tajo asociados al Horizonte de Cerámicas Lisas donde se ha evidenciado actividad metalúrgica, no pasan de ser útiles de pequeño tamaño y sencilla elaboración, fabricados posiblemente en el ámbito doméstico (Blasco y otros, 1995 a: 120). El Cerro del $\mathrm{Bu}$ no ha proporcionado hasta el momento restos que nos permitan afirmar la existencia de una producción local de elementos metálicos y menos aún de objetos que requieren cierta complejidad en su elaboración como es el caso de los puñales. Estas piezas se realizarían mediante fundición en molde bivalvo o en monovalvo bien perfilado seguido de un tratamiento de forja para rematar la pieza. Probablemente esta forja en frío afectaría sobre todo a los filos para después efectuar un recocido en la fragua con objeto de recristalizar el metal. Como vemos en su composición, el puñal está realizado en cobre pero se detecta un bajo contenido de estaño en la muestra (Rovira y otros, 1997: 377): (Tabla 1).

Se trata por lo tanto de un bronce pobre que hace dudar de la intencionalidad de esta aleación. El pequeño porcentaje de estaño apenas modifica las cualidades mecánicas del cobre, sin embargo, el hecho de que la mayoría de elementos de bronce en el mundo argárico se concen- tren sobre elementos de adorno hace que el interés por la nueva aleación se centre más en su valor como elemento de prestigio despreciando las cualidades físicas del nuevo metal. Tal vez, como afirma Montero (1994: 259), el valor de la aleación se manifestaba externamente en el color más dorado de las piezas de bronce, aunque este sólo es apreciable si el contenido de estaño supera el $10 \%$ por lo que esta explicación no es aplicable a la pieza del Bu.

La presencia de estaño en las mineralizaciones es capaz de proporcionar bronces naturales, sobre todo si tenemos en cuenta que en algunos casos en los enclaves cupríferos toledanos puede alcanzar el 15\% y a menudo intervalos del 3 y el 7 \% (Montero y otros, 1990). Sin embargo, la tecnología de reducción del mineral haría difícil la retención del estaño por las condiciones y la baja temperatura alcanzada en el proceso, inferior siempre a los $1000^{\circ} \mathrm{C}$ (Rovira y Montero, 1994: 167).

Su tipología, el hecho de haberse hallado en los niveles superiores y la presencia de estaño en su composición, aunque sea en un bajo porcentaje, parecen indicarnos su pertenencia a un momento avanzado del yacimiento, quizás ya en el Bronce Medio.

La presencia de un remache de plata indica que nos hallamos ante un elemento de prestigio, sobre todo si tenemos en cuenta la rareza de este metal que, aunque aparece con más frecuencia que el oro, está escasamente representado en la Meseta Sur. El empleo de remaches de plata es relativamente común en los puñales argáricos del Sureste, aunque también se han encontrado en la Espada de Puertollano (Ciudad Real) o en un brazal de arquero de la Morra del Quintanar (Albacete) (Montero, 2001: 285).

Los puñales aparecen desde el Campaniforme formando parte de ajuares funerarios pertenecientes a personajes destacados y son bastante frecuentes en todo el cuadrante Sureste peninsular durante la Edad del Bronce. En la Cuenca Media del Tajo no son comunes las armas como objetos de prestigio. Una de las escasas piezas de

Puñal Remaches

Inventario: CBU 86. 6. 7

No Análisis: A-4321.1

\begin{tabular}{|l|l|l|l|l|l|l|l|l|l|l|}
\hline $\mathrm{Fe}$ & $\mathrm{Ni}$ & $\mathrm{Cu}$ & $\mathrm{Zn}$ & $\mathrm{As}$ & $\mathrm{Ag}$ & $\mathrm{Sn}$ & $\mathrm{Sb}$ & $\mathrm{Pb}$ & $\mathrm{Bi}$ & $\mathrm{Au}$ \\
\hline 0.178 & nd & 96.52 & nd & nd & 0.017 & 2.521 & 0.090 & nd & nd & - \\
\hline
\end{tabular}




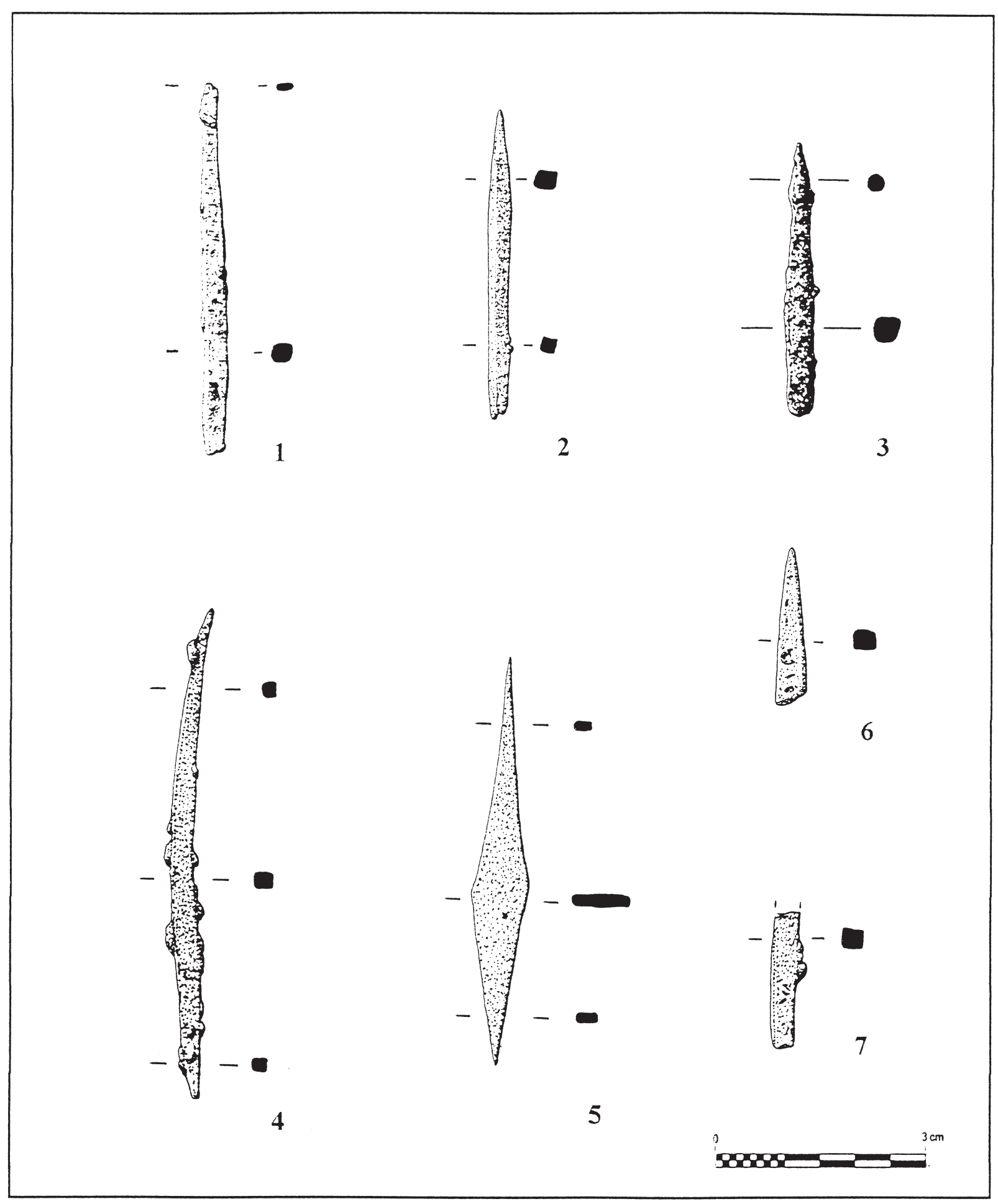

Figura 5.- Punzones de bronce. 
este tipo, encontrada en el área madrileña, es la espada de la Perla (Blasco y otros, 2001). Se trata de un hallazgo descontextualizado aunque posiblemente asociado a un hábitat del Bronce Clásico, al haberse encontrado muy cerca del yacimiento de Tejar del Sastre, por lo que podría ser sincrónica a la pieza del Bu. En este caso, la excepcionalidad de la pieza no está en la calidad del material en el que está fabricada, cobre arsenicado, sino en la cantidad de metal empleado ya que su peso es de 723 gramos, un volumen varias veces mayor que el total recuperado en nuestro yacimiento.

\section{Punzones}

Se han hallado un total de cinco punzones completos (Figura 5.1-4), además de dos puntas (Figuras 5.6 y 6.1) y dos fragmentos de vástago (Figuras 5.7 y 6.4) pertenecientes a piezas fragmentadas.

La mayoría es sección cuadrada o rectangular, excepto uno de ellos que presenta el extremo activo de sección circular (Figura 5.3). Los punzones con sección circular en la punta y cuadrada en la base son relativamente abundantes en la Edad del Bronce. Son habituales en el Campaniforme y su morfología parece estar en relación directa con el sistema de enmangue (Delibes, 1977: 112). Destaca también un punzón biapuntado de sección cuadrada (Figura 5. 4). Este tipo es uno de los elementos más comunes entre los objetos metálicos aparecidos en lugares de hábitat de la Edad del Bronce.

Los estudios metalográficos han proporcionado interesantes datos sobre el proceso de fabricación de estas piezas. Un primer paso sería la fundición a molde, seguido de la forja en frío del producto de esa fundición mediante martilleado para endurecer el metal y, en ocasiones, se fina-

\begin{tabular}{|l|l|}
\hline Tratamiento & Zona analizada \\
\hline Fundición + Forja en frío & Talón \\
\hline
\end{tabular}

Tabla 2 lizaría mediante un recocido térmico para eliminar la fragilidad de la pieza (Rovira y Gómez, 1994: 376). En el caso del Cerro del Bu, se realizó una metalografía de uno de los punzones que reveló el método de elaboración del mismo (Rovira y Gómez, 1994: 383): (Tabla 2).

Su composición fue analiaza por el proyecto Arqueometalúrgico de la Península Ibérica (Rovira y otros, 1997: 377): (Tabla 3).

El punzón está realizado en cobre arsenicado, dando un porcentaje de arsénico muy superior al resto de objetos de cobre hallados en la provincia, en los que se aprecia una baja concentración de este elemento quizá explicado por su escasa presencia en los minerales de cobre de los Montes de Toledo (Ruiz Taboada y Montero, 2000: 361). Los distintos investigadores admiten que estos cobres arsenicales son aleaciones accidentales debidas a la falta de control sobre las condiciones de trabajo metalúrgico.

Este tipo de punzones muchas veces conservan restos de acanaladuras en su base destinados a albergar el enmangue que se ha conservado en algún caso (Hernández y otros, 1994: 86).

Otro tipo de punzón hallado en el yacimiento es el doble losángico (Figura 5.5). Esta pieza, hallada en los niveles correspondientes a la segunda fase del yacimiento, tiene una longitud de $5,5 \mathrm{~cm}$ y una anchura máxima de $8 \mathrm{~mm}$. Presenta una forma romboidal y simétrica con dos extremos activos, bordes rectilíneos y sección rectangular. El ensanchamiento no se produce en la mitad de la pieza sino que está desplazado hacia uno de sus vértices por lo que uno de sus extremos está más aguzado que el otro.

Se podría encuadrar en el tipo I de la clasificación que plantea Roudil para los punzones losángicos del Languedóc oriental, siendo el tipo más común en el Sur de Francia (Roudil, 1972: 55). Este grupo, en el que se pueden situar la mayoría de los escasos ejemplares peninsulares, posee una longitud entre 30 y $60 \mathrm{~mm}$ y es, según algunos autores, el modelo más antiguo, anterior

Punzón sección rectangular

Inventario: CB $80 \quad$ No Análisis: AA0789

\begin{tabular}{|l|l|l|l|l|l|l|l|l|l|l|}
\hline $\mathrm{Fe}$ & $\mathrm{Ni}$ & $\mathrm{Cu}$ & $\mathrm{Zn}$ & $\mathrm{As}$ & $\mathrm{Ag}$ & $\mathrm{Sn}$ & $\mathrm{Sb}$ & $\mathrm{Pb}$ & $\mathrm{Bi}$ & $\mathrm{Au}$ \\
\hline 0.028 & 0.043 & 94.45 & 0.167 & 4.020 & 0.001 & 0.021 & 0.003 & $\mathrm{nd}$ & $\mathrm{Nd}$ & - \\
\hline
\end{tabular}


a otros evolucionados con perfiles cóncavos y secciones elípticas (Pérez Arrondo y López de Calle, 1986: 143).

Los punzones losángicos, también llamados punzones brújula o leznas losángicas (alènes losangiques), son piezas de influencia y origen centroeuropeo y que tienen en el Sureste de Francia una gran difusión asociadas a contextos de Bronce Antiguo y comienzos del Bronce Medio (Guilaine, 1972: 59). Estas piezas francesas están realizadas en bronce, al contrario de lo que parece ocurrir en Europa central y en la Península Ibérica (Roudil, 1972: 55).

Eran desconocidas en la Meseta hasta la publicación de los materiales del Castillo de Cardeñosa (Avila) donde aparecieron varias piezas con bordes tanto rectilíneos como cóncavos asociados a materiales pertenecientes al Bronce Antiguo (Naranjo González, 1984: 50). Se han encontrado, según Roudil (1972: 55), en el Argar y hay datos sobre hallazgos en Muñogalíndo (Ávila) y en el estuario del Tajo, en Villa Nova de Sao Pedro (Naranjo González, 1972: 64). En el caso de los hallados en el Noreste peninsular, se trata probablemente de piezas francesas importadas, lo que explicaría su presencia en los dos extremos del eje pirenaico (Pérez Arrondo y López de Calle, 1986: 159). Aparecen en la zona catalana asociados a cerámicas de transición Bronce Antiguo y Medio como los cuencos y tazas carenadas, los vasos hemiesféricos o las asas de apéndice de botón (Martín Cólliga, 1999: 145). Las analíticas realizadas sobre este tipo de punzones en la Península Ibérica, revelan su fabricación en cobre como es el caso de la pieza hallada en el dolmen Tarter del Tossal de Jovell, en Lérida, (Rovira y otros, 1997: 257) o la encontrada en el dolmen de Coll de Creus II, también en Lérida, que en un primer análisis publicado parecía estar realizado en bronce (Pérez Arrondo y López de Calle, 1987: 89), aunque recientes estudios han demostrado que se trata de cobre (Rovira y otros, 1997: 254). Sin embargo, no se descarta la existencia de ejemplares realizados en bronce. También existen punzones losángicos en hueso desde el Calcolítico a Bronce Final en el
Valle del Ebro (Rodanés, 1987: 82)

En cuanto a su función, Dechelette (1910: 341), a partir de hallazgos centroeuropeos, consideraba este tipo de objetos como punzones para realizar tatuajes y se utilizarían enmangados en madera o hueso. Roudil (1972: 55), aunque no está de acuerdo en este uso, sí les otorga cierto valor ritual al haberse hallado a menudo en ajuares funerarios y monumentos megalíticos del Calcolítico y Bronce Antiguo en los que no es frecuente hallar útiles.

El hallazgo de esta clase de punzones en la Meseta Sur, cuyo único ejemplar es el encontrado en el Cerro del $\mathrm{Bu}$, nos puede estar indicando contactos con los centros de producción de estos tipos situados en tierras transpirenaicas o al menos con el Noreste peninsular. En cualquier caso, al tratarse de objetos tan simples, la similitud formal puede ser el resultado de relaciones muy débiles o indirectas o incluso de fenómenos de convergencia (Fernández Miranda y otros, 1995: 59).

Por último, entre los elementos de cobre procedentes del Cerro del $\mathrm{Bu}$, destaca un fragmento de hoja de cuchillo (Figura 6.6) aunque hallado en un contexto poco claro junto a materiales del Hierro I.

\section{La cinta de oro (Figura 6.7)}

Se hallaron dos elementos de oro en el Cerro del $\mathrm{Bu}$. El primero de ellos no se ha podido localizar aunque parece que se trataba de una espiral que podría ser parte de un adorno (de Álvaro y Pereira, 1990: 208). El otro es una lamina o cintilla sin decoración de unos $6 \mathrm{~cm}$ de longitud, 2,5 $\mathrm{mm}$ de anchura y menos de $1 \mathrm{~mm}$ de grosor. El análisis espectrográfico realizado a la cinta por el proyecto Arqueometalurgia de la Península Ibérica reveló su composición (Rovira y otros, 1997: 377):*(Tabla 4).

Según Hernando (1983: 126) solamente dos hallazgos en la Península pueden ser integrados en este grupo de adornos:

- Una sepultura campaniforme en fosa plana en el Pago de la Peña (Villabuena

*Cinta Inventario: CBU 83.6. $24 \quad$ No Análisis: AA0787

\begin{tabular}{|l|l|l|l|l|l|l|l|l|l|l|}
\hline $\mathrm{Fe}$ & $\mathrm{Ni}$ & $\mathrm{Cu}$ & $\mathrm{Zn}$ & $\mathrm{As}$ & $\mathrm{Ag}$ & $\mathrm{Sn}$ & $\mathrm{Sb}$ & $\mathrm{Pb}$ & $\mathrm{Bi}$ & $\mathrm{Au}$ \\
\hline 0.411 & -- & nd & nd & $\mathrm{Nd}$ & 3.808 & 0.053 & 0.010 & $\mathrm{Nd}$ & nd & 94.95 \\
\hline
\end{tabular}




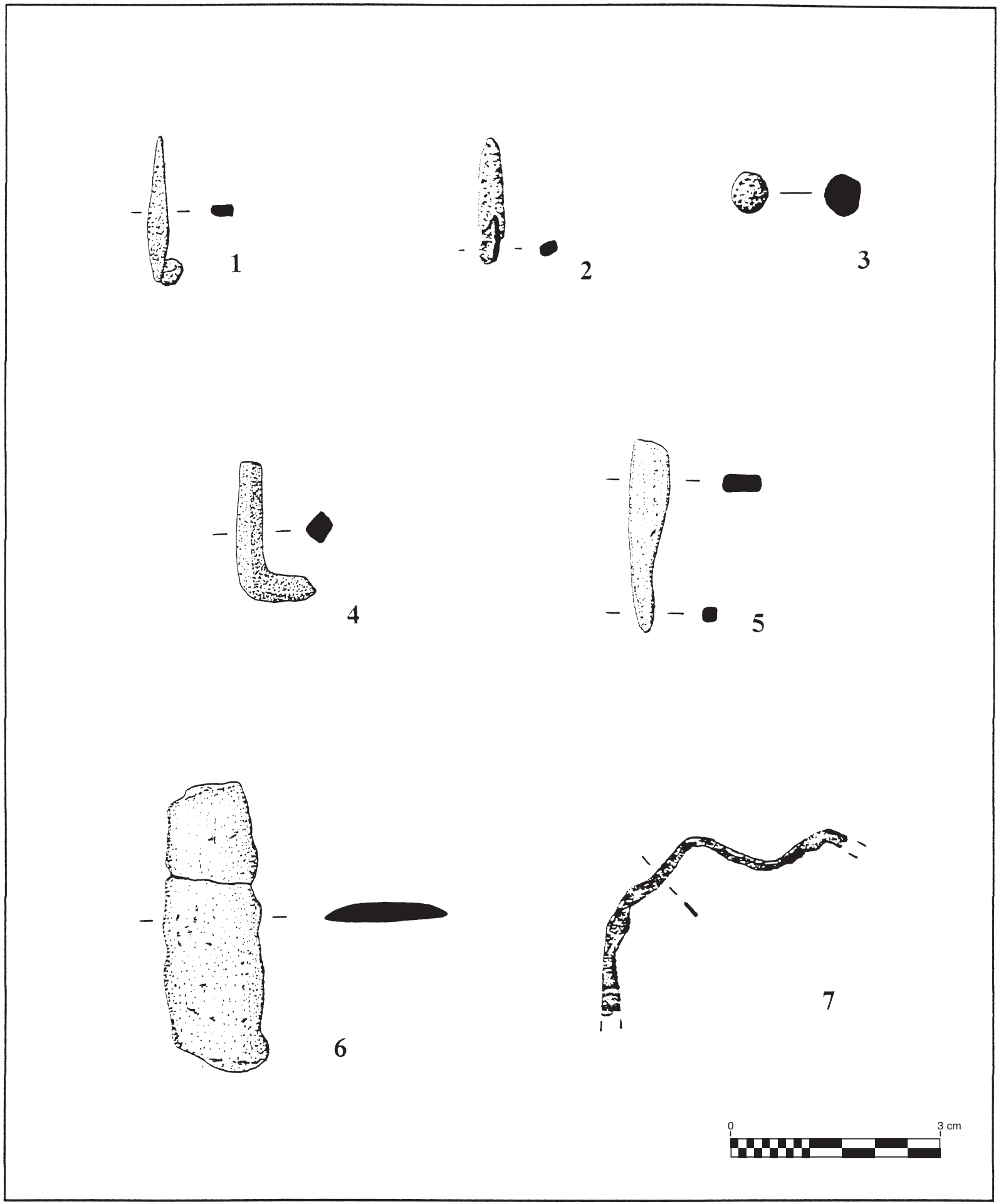

Figura 6.- Fragmento de hoja de cuchillo 
del Puente, Zamora) donde apareció una cintilla plegada en zigzags junto a un puñal de lengüeta, un brazal de arquero y botones de perforación en $\mathrm{V}$ (Maluquer, 1960: 127). La cinta de oro del cerro del Bu presenta una forma similar a la del Pago de la Peña y como ella parece haber estado plegada en zigzag, aunque es difícil asegurarlo ya que se encuentra muy deformada. Maluquer afirma que la cinta zamorana debió ser utilizada como un elemento de adorno pegado o incrustado en otro objeto como un brazalete o una diadema de cuero.

- El depósito de la finca La Paloma, en Pantoja, provincia de Toledo, situado a escasos 25 kilómetros al Norte del Cerro del $\mathrm{Bu}$, donde se encontraron dos alabardas con remaches, una sierra, un puñal de lengüeta, cuatro puntas palmela y una larga cinta de oro de entre 4 y $6 \mathrm{~cm}$ de anchura y rota en tres trozos que, según su descubridor, ataba el conjunto (Revuelta, 1980). Todo ello se encontraba en un vaso cerámico que pudo ser, según algunos autores, un vaso de carena media, cuello acampanado y borde exvasado (Carrobles y otros, 1994: 180). Las alabardas de tipo portugués o Carrapatas, de filiación irlandesa y posiblemente datables a finales del III milenio a. C., no son habituales en la Meseta y además de la pieza de Pantoja, únicamente se ha hallado un ejemplar en el valle del Manzanares (Blas Cortina, 1981) y otro en Villamiel (Toledo) (Ruiz Taboada y Montero, 2000: 360). Según Harbison serían de factura local pero evidenciarían una serie de influencias atlánticas que penetran en la Meseta a través del valle del Tajo. Este tipo de alabardas y el vaso carenado, con claros paralelos en los encontrados en el Cerro del $\mathrm{Bu}$, parecen apoyar una fecha avanzada del ocultamiento.

Posiblemente este tipo de cintas debieron ser elementos de adorno de mangos de puñal o bien piezas que decorarían objetos de carácter perecedero como diademas de cuero que no se han conservado (Hernándo Gonzalo, 1983: 128). Se asocian a contextos de Campaniforme tardío y están relacionados con un numeroso grupo de adornos simples que comienzan en este momento su desarrollo. El depósito de La Paloma, se podría encuadrar en una fase antigua del Horizonte de Cerámicas Lisas quizá sincrónica a ese Campaniforme tardío.

La metalurgia de oro tuvo gran desarrollo en el mundo campaniforme pero experimento una fuerte recesión durante el Bronce Antiguo y Medio, etapas en las se reduce significativamente el número de objetos realizados en este metal. El trabajo del oro debió realizarse desde un primer momento a partir del martilleo o batido de pepitas de metal halladas en estado nativo en placeres de los ríos (Hernando Gonzalo, 1983: 85). Sobre este aspecto, hay que mencionar que en el siglo XVI existen referencias de la existencia de antiguas explotaciones auríferas en las arenas del Tajo:

“...el limpisimo y claro Tajo de arenas doradas y llamase así por dos cosas, (...) la segunda porque los godos y romanos hallaban en el granos de oro, naturales de su suelo, densos, subtilisimos y lavados, de los mas excesivos quilates que se han visto (...) aunque es mas la costa quel provecho..." (Viñas y Paz, 1963: 500).

La cinta de oro se podría relacionar con la primera fase del asentamiento asociada posiblemente a los hallazgos campaniformes.

\section{La plata}

Además del remache de plata, se encontró una varilla realizada en este mismo material (Rovira y otros, 1997: 377): (Tabla 5).

La plata parece más ligada a los Bronces clásicos y aparece en el Argar y en el Bronce manchego muchas veces en contextos funerarios. Puede ser considerada como una innovación desarrollada desde el Bronce Antiguo relacionada en sus inicios con el aprovechamiento de la plata nativa o de cloruros de plata, ya que, hasta el momento, no existen pruebas objetivas del conocimiento de la técnica de copelación para

Varilla

Inventario: CBU 84. 6.11

No Análisis: AA1495

\begin{tabular}{|l|l|l|l|l|l|l|l|l|l|l|}
\hline $\mathrm{Fe}$ & $\mathrm{Ni}$ & $\mathrm{Cu}$ & $\mathrm{Zn}$ & $\mathrm{As}$ & $\mathrm{Ag}$ & $\mathrm{Sn}$ & $\mathrm{Sb}$ & $\mathrm{Pb}$ & $\mathrm{Bi}$ & $\mathrm{Au}$ \\
\hline $\mathrm{Nd}$ & -- & 1.256 & $\mathrm{nd}$ & $\mathrm{Nd}$ & 97.78 & $\mathrm{nd}$ & $\mathrm{Nd}$ & $\mathrm{nd}$ & $\mathrm{Nd}$ & nd \\
\hline
\end{tabular}


estas fechas (Montero, 1992: 195). La ausencia de contenidos significativos de plomo en los elementos de plata parece descartar el empleo de este procedimiento que permite la obtención de este metal a partir de galenas argentíferas (Montero, 1994: 265).

Otro inconveniente técnico que plantea el trabajo de la plata es la necesidad de un tratamiento térmico más cuidado que el del oro debido a su menor ductibilidad (Comendador Rey, 1999: 32). Los únicos hallazgos en la Meseta Sur de este metal se reducen a los remaches de un brazal de arenisca en uno de los enterramientos de la morra del Quintanar, dos brazaletes en tumbas infantiles del cerro de La Encantada y una Pulsera en el Cerro del Castillejo en Cuenca (Fernández Posse y otros, 1999: 229).

\section{Conclusiones}

En el Cerro del $\mathrm{Bu}$ no se han hallado elementos que nos permitan afirmar que se efectuaron labores de reducción del mineral por lo que no habría que descartar un comercio a pequeña escala de metal posiblemente en lingotes. La producción metalúrgica en los poblados durante este Bronce Antiguo y Medio en la Cuenca Media del Tajo pudo estar en manos de broncistas que trabajaban ocasionalmente cuando las necesidades lo requerían, aunque pudieron existir individuos especializados, una especie de metalúrgicos itinerantes dentro de ciertas regiones o comarcas que al mismo tiempo actuarían como buhoneros en la distribución de determinados productos (Ruiz Zapatero y Rovira i Port, 1994-1996: 35). Esta afirmación podría explicar la presencia de objetos de procedencia o tipología foránea como el punzón losángico. La ubicación del yacimiento, situado en un nudo de comunicaciones por la presencia del vado del Tajo, facilita el establecimiento de contactos con otras áreas. La pervivencia del asentamiento durante varios siglos pudo estar basada en el control de esas vías de comunicación que podría dar lugar al surgimiento de una pequeña elite que desarrollaría contactos con poblaciones o grupos cercanos a través de los cuales se podría surtir de elementos de prestigio.

Aunque se trataría de una sociedad relativamente igualitaria, no se descarta la existencia de una clase dirigente en la que el metal debió jugar un papel importante. Estas elites no se sustentarían mediante el control de la extracción de mineral o la elaboración de metal sino sobre la producción agrícola o ganadera que sería sin duda la base económica del yacimiento además de ese posible dominio sobre el vado del río. La metalurgia no sería una actividad de especialistas a tiempo completo, sino complementaria, esporádica, que no requeriría grandes inversiones de trabajo en las minas (Montero, 1992: 207). Sin embargo, el metal, por su escasez, debió ser un elemento de estatus y distinción social pese a que se utilizase mayoritariamente en la confección de objetos funcionales. En la Cuenca Media del Tajo y en el conjunto de la Meseta Sur, destaca la inexistencia de adornos de metal como anillos, pendientes, brazaletes o cuentas que sí son habituales en una sociedad jerarquizada como la argárica. Los nuevos tipos de puñal, las aleaciones y los adornos son indicadores de competitividad social y se desarrollan con mayor rapidez dependiendo de la necesidad social de diferenciación y exaltación de las elites de un grupo (Rovira y Montero, 1994: 170; Blasco y Lucas, 2002: 312).

Muñóz (2001: 135) afirma que las desigualdades en los grupos que desde el Calcolítico poblaron el Valle del Tajo se harían cada vez más grandes y se plasmarían en la posesión de determinados objetos con connotaciones prestigiosas, la jerarquización del hábitat y la exclusividad del mundo funerario. Esos objetos de factura compleja o foránea, o elaborados con materias primas exóticas, distinguirían a los usuarios frente al resto de su comunidad y frente a otras comunidades. El poder y el liderazgo de estos grupos surgiría del control y la acumulación de recursos, en especial suelos agrícolas, pastos húmedos y grandes rebaños junto con el control de los pasos y vías de comunicación y otros elementos como la sal (Muñoz, 2001: 135)

Los objetos de prestigio, y especialmente algunos metálicos como las espadas, se han llegado a entender como una evidencia de competencia entre elites que se desarrolla desde el Bronce Antiguo ligada a los circuitos de intercambio, la adquisición de metales como la plata y posiblemente la búsqueda de estaño (Blasco y otros, 2001: 73). Sin embargo, en el registro arqueológico no se manifiesta esa importancia del metal y no es posible afirmar que, durante la segunda mitad del III milenio y la primera del II milenio cal. B. C. en la Cuenca Media del Tajo, el metal fuese el causante de una transformación social.

\section{Bibliografía}

Aubet, M. E.; Serna, M. R. (1981): "Una sepultura de la Edad del Bronce en Setefilla". Trabajos de Prehistoria, 38: 225-256.

Blance, B. (1971): Die anfänge der metallurgie auf der Iberischen Halbinsel. SAM, 4. Berlín. 
Blas Cortina, M. A. (1981): "Una alabarda procedente del Valle del Manzanares". Zephyrus, XXXIIXXXIII: 157-166.

Blasco, M. C.; BARRIO, J. (1986): "Excavaciones de dos nuevos asentamientos Prehistóricos en Getafe (Madrid)". Noticiario Arqueológico Hispánico, 27: 75142.

Blasco, M. C.; LuCAS, M. R. (2002): "Lectura social de la metalurgia del bronce en el Noroeste de la Meseta Sur". Primer simposio de arqueología de Guadalajara: 309-334.

Blasco, M. C.; Rovira, S. (1992-1993): "La metalurgia del Cobre y del Bronce en la región de Madrid". Tabona, VIII, T. II: 397-415.

Blasco, M. C.; Baena, J.; Lucas, M. R.; Carrión, E. (2001): "La espada de la Perla. Una pieza excepcional conocida a través de la obra de José Pérez de Barradas". Estudios de Prehistoria y Arqueología madrileñas, 11: 69-85.

Blasco, M. C.; Calle Pardo, J.; Sánchez Capilla, M. L. (1995 a): "Contribución al estudio de la metalurgia del bronce en el Alto Tajo y su marco cultural.”. Homenaje a Dra. Milagro Gil-Mascarell Boscá. Extremadura Arqueológica, V: 115-128.

Blasco, M. C.; Calle Pardo, J.; Sánchez Capilla, M. L. (1995 b): "Fecha de C14 de la fase Protocogotas I del yacimiento del Caserío de Perales del Río". Cuadernos de Prehistoria y Arqueología de la Universidad Autónoma de Madrid, 22: 83-99.

Carrobles, J.; Muñoz, K.; Rodríguez, S. (1994): "Poblamiento durante la Edad del Bronce en la Cuenca Media del río Tajo". La Edad del Bronce en Castilla-La Mancha. Toledo: 173-200.

Castro Martínez, P. V.; Lull, V.; Micó, R. (1996): Cronología de la Prehistoria Reciente de la Península Ibérica y Balerares (c. 2800-900 cal ANE). BAR International Series, 652. Oxford.

Comendador Rey, B. (1999): "Noroeste" en Delibes de Castro, G.; Montero Ruiz, I. (Coord): Las primeras etapas metalúrgicas en la Península Ibérica, II. Estudios regionales. Madrid: 9-39.

De Álvaro, E.; Pereira Sieso, J. (1990): "El Cerro del $\mathrm{Bu}$ (Toledo)". Actas del Primer Congreso de Arqueología de la Provincia de Toledo. Toledo: 199-213.

Déchelette, J. (1910): Manuel d'archéologie préhisotirque, celtique et gallo-romaine. T. II. Archéeologie celtique ou protohistorique. París.

Delibes de Castro, G. (1977): El vaso campaniforme en la Meseta Norte española. Valladolid.

Fernández Miranda, M.; Montero, I.; Rovira, S.
(1995): "Los primeros objetos de Bronce en el Occidente de Europa". Trabajos de Prehistoria, 52 (I): 57-69.

Fernández Posse, M. ${ }^{a}$ D.; Martín, C.; Montero, I. (1999): "La Meseta Sur" en Delibes de Castro, G.; Montero Ruiz, I. (Coord): Las primeras etapas metalúrgicas en la Península Ibérica, II. Estudios regionales. Madrid: 217-240.

Guilaine, J. (1972): L’age du bronze en Languedoc Occidental, Rousillon, Ariège. Mémoires de la Société Préhistorique Française, 9. París.

Hernández Pérez, M.; Simón García, J. L. ; López Mira, J. A. (1994): Agua y Poder. El Cerro de El Cuchillo (Almansa, Albacete). Toledo.

Hernándo GonZalo, A. (1983): "La orfebrería durante el Calcolítico y el Bronce Antiguo en la Península Ibérica". Trabajos de Prehistoria, 40: 86-138.

MaluQuer de Montes, J. (1960): "Nuevos hallazgos de la cultura del vaso campaniforme en la Meseta" Zephyrus, XI: 119-130.

Martín Colliga, A.; Gallart Fernández, J.; Rovira Hortalá, C.; Mata-Perelló, J. M. (1999): "Nordeste" en Delibes de Castro, G.; Montero Ruiz, I. (Coord): Las primeras etapas metalúrgicas en la Península Ibérica, II. Estudios regionales. Madrid: 115-177.

Montero Ruiz, I. (1992): “ La actividad metalúrgica en la Edad del Bronce del sudeste de la Península Ibérica: tecnología e interpretación cultural". Trabajos de Prehistoria, 49: 189-215.

Montero Ruiz, I. (1994): El origen de la metalurgia en el Sur de la Península Ibérica. Almería.

Montero Ruiz, I. (2001): "Estudios sobre metalurgia antigua en la Provincia de Toledo" II Congreso de arqueología de la Provincia de Toledo, 1. Toledo: 275301.

Montero Ruiz, I.; Rodríguez Montero, S; Rojas Rodríguez-Malo, J. M. (1990): Arqueometalurgia de la Provincia de Toledo: Minería y recursos minerales de cobre. Toledo.

Moreno Onorato, A. (2000): "La metalurgia de Peñalosa" en Contrerás Cortés, F. (Coord.): Proyecto Peñalosa. Análisis histórico de las comunidades de la Edad del Bronce del piedemonte meridional de Sierra Morena y depresión Linares-Bailén. Sevilla: 167-222.

Naranjo González, C. (1984): “ El Castillo de Cardeñosa. Un yacimiento de los inicios de la Edad del Bronce en la Sierra de Ávila (Excavaciones reealizadas por J. Cabré en 1931)", Noticiario Arqueológico Hispánico, 19: 35-84. 
Pérez Arrondo, C. L.; lópez de Calle, C. (1986): Aportaciones al Estudio de las culturas eneolíticas en el Valle del Ebro, II. Los orígenes de la Metalurgia. Logroño.

Quero Castro, S. (1982): "El poblado del Bronce Medio de Tejar del Sastre". Estudios de Prehistoria y Arqueología madrileñas, 1: 185-247.

Revuelta Turbino, M. (1980): "Los hallazgos de Pantoja en el Museo de Santa Cruz". Toletum, 10: 952.

Rodanés Vicente, J. M. (1987): La industria ósea prehistórica en el Valle del Ebro. Zaragoza.

Rojas Rodríguez Malo, J. M; Rodrígez Montero, S. (1990): "El Guijo: Aportación al Estudio del calcolítico y la Edad del Bronce en la Cuenca Media del Tajo". Actas del I Congreso de Arqueología de la Provincia de Toledo. Toledo: 163-198.

RoudiL, J. L. (1972): L'age du bronze en Languedoc Oriental. Mémoires de la Société Préhistorique Française, 10. París.
Rovira, S.; Gómez Ramos, P. (1994): "Punzones y varillas metálicas en la Prehistoria reciente española: un estudio tecnológico". Espacio, Tiempo y Forma. Serie I, Prehistoria y Arqueología. T. 7: 371-402.

Rovira, S.; Montero, I. (1994): "Metalurgia campaniforme y de la Edad del Bronce en la Comunidad de Madrid", en Blasco (ed), El horizonte campaniforme en la región de Madrid en el centenario de Ciempozuelos. Madrid: 137-171.

Rovira, S.; Montero I.; Consuegra, S. (1997): Las primeras etapas metalúrgicas en la Península Ibérica, I. Análisis de materiales. Madrid.

Ruiz Taboada, A.; Montero, I. (2000): "The pattern of use of stone and copper in central spain during the Bronce Age" European Journal of Archaeology, Vol. 3 (3): 350-369

VIÑAS, C.; PAZ, R. (1963): Relaciones historico-geográficoestadísticas de los pueblos de España hechas por iniciativa de Felipe II. Reino de Toledo. Tercera parte. Madrid. 